\title{
Selection by Parts: 'Selection in Two Episodes' in Evolutionary Algorithms
}

\author{
Ambedkar Dukkipati and M. Narasimha Murty \\ Department of Computer Science and Automation \\ Indian Institute of Science \\ Bangalore-560012, India \\ Email: \{ambedkar, mnm\}@csa.iisc.ernet .in
}

\begin{abstract}
Natural selection is the central concept of Darwinian evolution and hence selection is central for evolutionary computation. Naive models of evolution define natural selection as a process which brings in differential reproductive capabilities in organisms of a population, and hence, evolutionary algorithms implement selection by differential reproduction: the fittest members of the population are reproduced preferentially at the expense of the less fit members of the population. Formal models in evolutionary biology often subdivide selection into components called 'episodes of selection' to capture the different complex mechanisms of nature by which Darwinian evolution can occur. In this paper we introduce the concept of "episodes of selection' in evolutionary computation by means of "A Conceptual Evolutionary model"( $A C E$-model), This model captures selection in two episodes in two phases of evolutionary cycle. Here we give formal description of ACE-model, in which one can mechanize the two phases in different possible ways. To demonstrate the concept of episodes of selection we propose evolutionary algorithms based on ACE-model, by giving simple mechanisms for implementation of two phases. Finally we discuss the importance of introducing episodes of selection in evolutionary algorithms by simulations of proposed evolutionary algorithms for function optimization.
\end{abstract}

\section{INTRODUCTION}

Evolutionary algorithms mimic Darwinian evolution i.e., "evolution by natural selection" since Darwinian evolution is intrinsically a robust search and optimization mechanism [4]. Evolutionary computation uses the metaphor of mapping "problem solving" onto a simple model of evolution as shown below [6]:

\begin{tabular}{ccc}
\hline \hline Evolution & $\Longleftrightarrow$ & Problem Solving \\
\hline environment & $\longleftrightarrow$ & problem \\
organism & $\longleftrightarrow$ & candidate solution \\
fitness & $\longleftrightarrow$ & quality \\
\hline \hline
\end{tabular}

In any kind of evolutionary algorithm, selection forms an important operator due to its independence of the actual search space. Current evolutionary algorithms propose several selection mechanisms, by viewing natural selection as a process which brings in differential reproductive capabilities in a population. But the formal models of Darwinian evolution capture the complex mechanisms of natural selection by dividing it into components which are called "episodes of selection". Two such important episodes are viability selection and fertility selection, both of which occur in an evolutionary cycle. Viability selection results due to differences in survival capabilities and fertility selection results due to differences in reproductive capabilities of organisms in a population respectively.

The concept of 'survival mechanism' is first captured in evolutionary algorithms by "A Conceptual Evolutionary model"'(ACE-mode1) [2]. The modified and generalized ACEmodel is given in [3] which mechanizes survival by Malthusian principle of population.

Now, in this paper we introduce two episodes of selection into evolutionary computation by means of ACE-model. We discuss essential biological concepts which motivated ACEmodel in Section II, and in Section III we give formal presentation of ACE-model. We propose a simple mechanism to construct evolutionary algorithms on ACE-model in Section IV and discuss the importance of various parameters through simulation studies in Section V.

\section{DARWINIAN EVOLUTION}

\section{A. Evolution by Natural Selection}

Evolution is a process that results in heritable changes in a population spread over many generations. Darwin was the first to explain the process of evolution by natural selection. Darwin used 'fitness' in evolutionary sense and then Spencer phrased natural selection as "survival of fittest" [10]. In this section we briefly discuss fitness as a concept.

\section{B. The Concept of Fitness}

In evolutionary biology the term 'fitness' was used long before an attempt was made to quantify it. In the 'Darwinian evolution'(evolution by natural selection), fitness was used to 
describe an organism's vigor, or the degree to which an organism 'fits' into its environment i.e., literally it represents the survival capability of organisms in terms of the struggle between organisms for limited resources (biotic competition) and the struggle against features such as drought, of the nonliving physical environment (abiotic competition).

While attempting to quantify fitness biologists started viewing natural selection as an abstract process which brings in evolutionary change, rather than as a specific process. This led them to define fitness in terms of reproductive success of organisms. In mathematical terms it is defined as the expected number of offspring an organism(which has survived) can produce for next generation. Fitness is also defined in terms of expected number of offspring that will survive but [11] warns about measuring fitness 'across the generations'.

\section{Selection Episodes and Fitness Components}

Even though fitness, defined as the expected number of offspring, captures abstract, simple and wide perspective of evolutionary change, it ignores some of the finer aspects of evolutionary process which may bring in different kinds of evolutionary changes. These finer aspects can be captured by dividing natural selection into components called episodes of selection [11].

Not only selection has an impact on the traits that determine how likely it is for an organism to survive from the egg stage to adulthood, but it equally has an impact on the traits that determine how successful an adult organism is likely to be in having offspring [9]. Hence we can identify two important episodes of selection viz., viability selection and fertility selection. The two life-history parameters of an organismi.e., viability and fertility need to be defined at this stage. Viability of an organism is the probability that organism at the egg stage will reach adulthood, and fertility of an organism is the expected number of offspring that the adult organism will have. Hence, viability selection causes differences in viabilities of organisms and, fertility selection causes differences in fertilities of organisms in a population. With respect to the two episodes of selection:viability selection(occurs from egg stage to reproductive-age) and fertility selection(occurs from reproductive-age to its death) we can view viability and fertility as the two components of fitness.

Note that fitness can have several components - though viability and fertility are the important components of selection in 'organismic evolution' - for each possible episode of selection and these are defined to be 'multiplicative'. "Natural selection" results from variance in all the fitness components(except variance in male mating success due to malemale competition for females and/or female choice of particular males, which is called sexual selection). For more details refer to the formal treatment given in [11].

\section{ACE-MODEL}

\section{A. Approach}

ACE-model is proposed to be simple and yet it captures the essential features of modem evolutionary perspectives as discussed above, in an evolutionary computation framework. Here, we briefly give the important features of ACE-model and in the next section we give a formal description of the same.

In ACE-model we capture two episodes of natural selection, in two phases of an 'evolutionary cycle'. For the sake of defining selection in two episodes, we divide lifespan (equivalent to one cycle of evolutionary process) of an organism into two phases: pre-reproductivephase and post-reproductive phase. The parameter reproductive-age is defined as the age at which all the organisms reach adulthood to reproduce. We assume reproductive-age to be constant for all the organisms under consideration, because, we are dealing with micro-evolution in which all the organisms belong to the same species. Hence we call reproductive-age as a species parameter. We introduce one more species-parameter called meanfertility which is defined as the average number of offspring an organism produces in its life time. This allows us to characterize reproductive phase easily.

ACE-model has two structural components: Population and Environment. In this paper we give only formal definitions of these; for the conceptual discussions see [2] and [3]. Viability selection takes place in the pre-reproductive phase and fertility selection takes place in the post-reproductive phase which form the two phases of evolutionary cycle. The prereproductive phase and post-reproductivephase are called survivalphase and reproductivephase respectively.

\section{B. Definitions}

Basic definitions: Here we list out basic notation:

$\boldsymbol{\Omega}$ is search-space which represents set of all possible organisms corresponding to particular species.

$w \in \Omega$ is an organism.

$\mathcal{P}$ is a set of all non empty multi-subsets of $\Omega$ and it represents set of all possible populations.

$\boldsymbol{\tau}=\{0,1,2, \ldots\}$ is index set to represent the generations (evolutionary cycle).

Species parameters: We have two species parameters:

$R \in[0,1]$ is reproductive-ageat which all the organisms reach adult-hood to participate in reproduction.

$\overrightarrow{\boldsymbol{\xi}} \in \mathcal{Z}^{+}$is mean-fertility defined as average number of offspring each organism can give in its lifetime.

Life span: Life span of an organism corresponds to one single evolutionary cycle (generation)(see Fig. 1). At generation $t \in \tau$, any instance is denoted by $t_{[x]}$ or $t[x]$ (we use both the forms depending upon conve- 
nience) where $x \in[0,1]$. Two such important instances are population at $t_{[0]}$ which is called offspring population $\left(P\left(t_{[0]}\right)\right.$ or $\left.P(t[0])\right)$ and population at $t_{[R]}$ which is called adult population $\left(P\left(t_{[R]}\right)\right.$ or $\left.P(t[R])\right)$. Note that $P\left(t_{[1]}\right) \equiv P\left((t+1)_{[0]}\right)$ i.e., population at $t_{[1]}$ is equivalent to offspring-population at generation $t+1$, since we are using non overlapping generations. Also unless it is specifically mentioned, by saying population at time $t$ we mean adult-population at time $t$ and write this as $P(t)$.

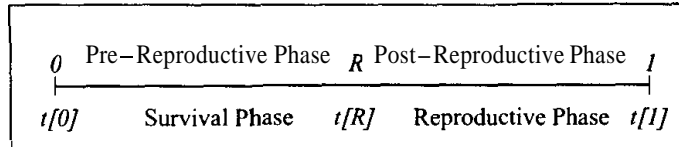

Fig. 1. The two phases of evolutionary cycle at generation $t$

Life-history parameters: We have two life-history parameters:

$\nu$ is viability of an organism, defined as the probability that it reaches reproductive-age.

$\boldsymbol{\xi}$ is fertility of an organism; it is a random variable which denotes the number of offspring it is going to give.

Relationships between the organisms: Relationships among the organisms of same population or different populations differ by time:

$\vdash \subseteq \Omega \times \Omega$ represents the relation 'immediate descent' defined as $\omega_{i}, \omega_{j} \in \Omega$ and $\omega_{i} \vdash \omega_{j}$ if and only if $\omega_{i}$ "causally produces" $\omega_{j}$ [5]. $\omega_{j}$ is said to be immediate-descent of $\omega_{i}$. The relation $\mathbf{f}$ is asymmetric, irreflexive and nontransitive relation. (For abstract definition see [7])

$\vDash \subseteq \Omega \times \Omega$ represents the relation 'descent' defined as $\omega_{i}, \omega_{j} \in \Omega$ and $\omega_{i} \models \omega_{j}$ if and only if $\omega_{i} \vdash \omega_{j}$ or $\exists\left\{\omega_{k}\right\}_{k=1}^{m}$ c $\Omega, m<\infty$ such that $\omega_{i} \vdash \omega_{1} \vdash$ $\omega_{2} \ldots \omega_{m} \vdash \omega_{j} . \omega_{j}$ is said to be descent of $\omega_{i}$. The relation $\models$ is asymmetric, irreflexive and transitive relation.

\section{System}

Structure: ACE-model has two structural units population $(P(t))$ and environment $\left(\mathcal{E}^{t}\right)$ defined as follows [2]:

$\boldsymbol{P}(t)$ is population at time $t$ and $P(t)=\left\{\omega_{k}\right\}_{k=1}^{N_{\mathrm{t}}}$ a non-empty multi-set of organisms

- $\omega_{k}$ is an organism $\forall k=1 \ldots N_{t}$

- $N_{t}$ is population size at time $t$

$\mathcal{E}^{t}$ is environment at time $t . \mathcal{E}^{t}=\left(f: \Omega \rightarrow \Re, K^{t}\right)$

- $f: \Omega \rightarrow \Re$ isfitness landscape

- $K^{t}$ is carrying-capacity of the environment, which is an 'ability of the environment' - the number of adult organisms that the environment can sustain
Process: The evolutionary process in ACE-model consists of survival phase $(\Psi)$ followed by reproductive phase $(\Lambda)$ or vice versa defined as follows:

$\Psi: P \rightarrow \mathcal{P}$ and $\Psi\left(P\left(t_{[0]}\right)\right)=P\left(t_{[R]}\right)$
$\quad$ is characterized by

$$
\operatorname{Prob}\left\{\omega \in P\left(t_{[R]}\right)\right\}=\nu(\omega) \quad \forall \omega \in P\left(t_{[0]}\right)
$$

where $\nu(\omega)$ is viability of organism $\mathrm{w}$. Note that $P\left(t_{[0]}\right) \supseteq P\left(t_{[R]}\right)$ (multi sub set).

A: $P \rightarrow \mathcal{P}$ and $\Lambda\left(P\left(t_{[R]}\right)\right)=P\left(t_{[1]}\right)$

is characterized by

$$
\begin{gathered}
\operatorname{Prob}\left\{\exists\left\{\omega_{i}\right\}_{i=1}^{\xi} \operatorname{cP}\left(t_{[1]}\right) \ni \mathrm{w} \vdash \omega_{i} \forall i\right\}= \\
\operatorname{Prob}\{\xi(\omega)=\xi\} \quad \forall \omega \in P\left(t_{[R]}\right)
\end{gathered}
$$

where [(instance of random variable $[(w))$ is the fertility of organism $\mathrm{w}$.

Note that $N_{t_{[R]}} \leq N_{t_{[1]}}$.

\section{Mechanization of Survival and Reproductive Phases}

The mechanism of survival phase and reproductive phase in ACE-model depends upon the methods by which we quantify viability and fertility of organisms in a population respectively. One can specify these mechanisms "functionally" or "operationally". By operational we mean that we give steps to perform survival or reproductive phases without quantifying viability and fertility explicitly.

Usually viability of an organism in the population depends upon: its fitness, fitness of other organisms in the population, and carrying capacity.

To mechanize reproductive phase one has to specify a method to assign fertilities. The actual mating process i.e., selecting a mate and giving birth is same as in the usual evolutionary algorithms. Note that fertility is a random variable and has a probability distribution. Also we would like to clarify the confusion that may arise between species parameter mean-fertility $(<)$ and mean of fertility of an organism in a population. $\bar{\xi}$ denotes the average number of offspring any organism in consideration produces, and this is introduced to give an idea about fertility distribution of organism. Mean fertility or expectation of ran+-variable $\quad[(w)$ on the other hand is denoted by $E \xi(\omega)$ or $\xi(\omega)$ and we prefer first notation to avoid confusion. Further, the distribution of $\xi(\omega)$ depends upon $\bar{\xi}$. One can assume particular type of distributions (for example Gaussian distribution) to quantify fertilities.

We discuss specific mechanisms in the next section.

\section{E. Evolutionary algorithms based on ACE-model (ACE- algorithms)}

Fitness landscape $(f: \Omega \rightarrow \Re$ ) as the objective function and $\operatorname{organism}(\omega)$ as a candidate solution, different evolutionary al- 
gorithms can be developed based on ACE-model by giving different methods to quantify viability and fertility such that they are proportional to fitness values of $\operatorname{organisms}(f(w))$. Such an evolutionary algorithm consists of random initialization of population $P(0)$ followed by the process $P(t+\mathbf{1})=$ $\Psi(\Lambda(P(t)))$, with a stop criterion. Note that each evolutionary algorithm with specific $\mathbf{A}$ and $\Psi$ depends upon $\mathrm{R}, \bar{\xi}$, $K^{t} \forall t \in \tau$.

\section{F. Advantages of ACE-algorithms}

ACE-model is.rich enough to serve as a basic model for evolutionary algorithms. ACE-model captures two - conceptually and mechanically different - selection mechanisms namely 'selection without replacement'(viability selection) and 'selection with replacement'(fertility selection) in the two phases of evolutionary cycle, survival phase and reproductive phase respectively. It offers various parameters by which evolutionary algorithms can control the selection intensities.

ACE-algorithms can explore the following parameters:

$K^{t} \forall t \in \tau$ : An evolutionary algorithm by giving 'control mechanism' for $K^{t}$ (online or offline) can control selection intensity directly.

$R$ : An evolutionary algorithm can choose different intensities of these selection mechanisms by choosing $R \in$ $[0,1]$.

$\bar{\xi}$ : By choosing $\bar{\xi}$ an evolutionary algorithm can decide upon how much diversity it can introduce in the population.

\section{MALTHUS-SPENCER EVOLUTIONARY ALGORITHM (MSEA)}

\section{A. Survival Phase by Malthusian Criteria}

Malthusian principle of population played a crucial role in evolutionary biology, and Darwin himself mechanized concept of natural selection based on it. [3] gives mechanism (operationally) for survival phase by Malthusian criteria and proposes Malthusian evolutionary algorithm. We can specify this functionally as:

Let $P\left(t_{[0]}\right)=\left\{\omega_{i}\right\}_{i=1}^{N_{t[0]}}$ be offspring-population. Let $\left\{f_{i}\right\}_{i=1}^{N_{t[0]}}$ be corresponding fitness values and $f_{i_{1}} \geq f_{i_{2}} \ldots \geq f_{i_{N_{t}[0]}}$ be the ordering of the fitness values (not necessarily distinct). Now according to Malthusian criteria we assign viabilities as:

$$
\nu\left(\omega_{i}\right)= \begin{cases}1 & \text { if } f\left(\omega_{i}\right)>f_{i^{t}} \\ 0 & \text { otherwise }\end{cases}
$$

where $K^{t}$ is carrying capacity.

In this mechanism $K^{t}$ determines the intensity of viable selection [3].

\section{B. Reproductive Phase based on Spencer's Hypothesis}

To mechanize the reproductive phase in ACE-model we have to give a method to assign probability distribution to fertility which is a random variable. Simplest distribution we can think of to represent the distribution of fertility $\xi(\omega)$ is Gaussian. In this paper we have considered Gaussian distribution with unit variance and the expectation that depends on fitness of organism. We discuss such a mechanism below.

There are several interpretations for fertility in evolutionary biology; one of them is due to Spencer. He interpreted fertility in terms of 'survival' of organisms. According to him: "fitness is capacity to survive, and survival was a pre-requisite for reproductive success" [13. That means fertility of an organism in a population depends upon how long it can survive after its reproductive age. Here we mechanize the reproductive phase based on this interpretation of fertility.

To incorporate this idea into ACE-model we have following hypothesis: "the variance in fertility of organisms is proportional to span of reproductive period" i.e., more the reproductive period, more the organism has to participate in the struggle to survive. This brings in an increased difference in fertilities of organism. We capture this as:

Let 6 be variance that can occur from meanfertility $(\bar{\xi})$. The only parameter we have to quantify is expectation of fertility $E \xi(\omega)$. Hence

$$
E \xi(\omega) \in[\bar{\xi}-6, \bar{\xi}+\delta] \text { for any organism } \omega
$$

Now we define variance 6 according to equation:

$$
\delta=\bar{\xi}(1-R)
$$

note that $0 \leq 6 \leq \vec{\xi}$ since $0 \leq R \leq 1$.

Now we assign fertilities to each of the organism in the adult-population $P\left(t_{[R]}\right)=\left\{\omega_{i}\right\}_{i=1}^{N_{t[R]}}$.

The proportionate relative fitness values are calculated as:

$$
f^{\prime}\left(\omega_{i}\right)=\frac{f\left(\omega_{i}\right)}{\sum_{j=1}^{N_{t[R]}} f\left(\omega_{j}\right)} \forall i=1 \ldots N_{t[R]}
$$

Then we define $E \xi\left(\omega_{i}\right)$ as:

$$
E \xi\left(\omega_{i}\right)=(\bar{\xi}-6)+2 \delta f^{\prime}\left(\omega_{i}\right) \forall i=1 \ldots N_{t[R]}
$$

which shows assigning of $E \xi$ to each of organism in the interval $[\vec{\xi}-\delta, \bar{\xi}+\delta]$ depending upon their proportionate relative fitness values.

This gives a simple mechanism for the reproductive phase. Note that $R$ determines the intensity of fertility selection. 


\section{Algorithm}

Here we give evolutionary algorithm with survival phase mechanized by Malthusian criteria and reproductive phase by Spencer hypothesis.

Algorithm for optimization of function $f: \Omega \rightarrow \Re$ is:

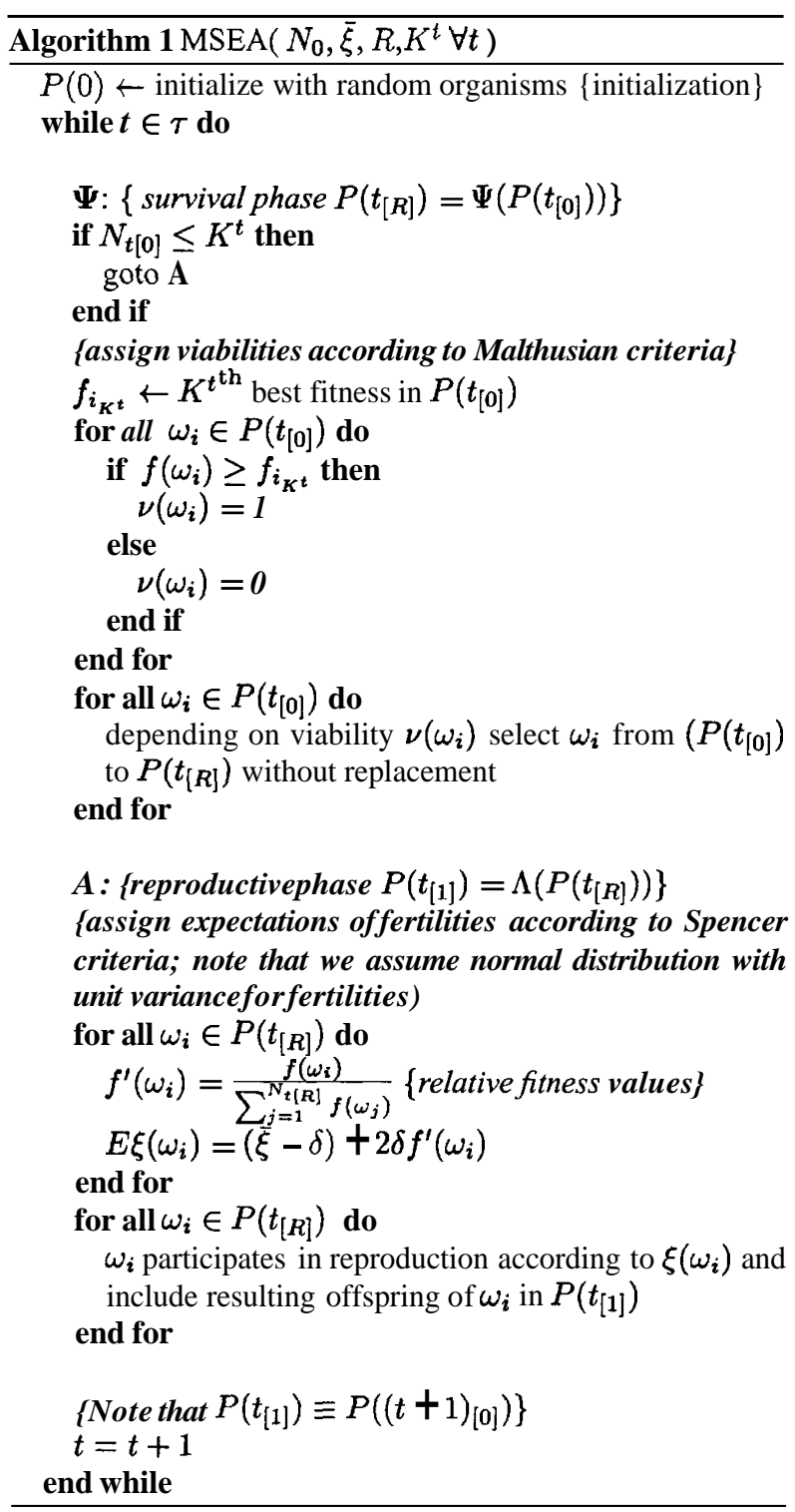

\section{SIMULATIONS}

We discuss the simulations conducted to study the evolutionary algorithms developed on ACE-model here. We study multi-variable function optimization in genetic algorithms framework. Specifically, we use the following functions [8]:

- Rastrigin's function $f_{6}(\vec{x})=n A+\sum_{i=1}^{n} x_{i}^{2}-A \cos \left(2 \pi x_{i}\right)$ where $A=10 ;-5.12 \leq x_{i} \leq 5.12$

- Ackley's function

$$
\begin{aligned}
& f_{9}(\vec{x})=-20 \exp \left(-0.2 \sqrt{\frac{1}{n} \sum_{i=1}^{n} x_{i}^{2}}\right) \\
& -\exp \left(\frac{1}{n} \sum_{i=1} n \cos \left(2 \pi x_{i}\right)\right)+20+e \\
& \text { where }-\mathbf{3 0} \leq x_{i} \leq \mathbf{3 0}
\end{aligned}
$$

We simulate proposed algorithms with different values of parameters $\bar{\xi}$ and $R$.

The following parameter values have been used in all the experiments :

- Each $x_{i}$ in $f_{6}$ and $f_{9}$ is encoded with 5 bits and $n=30$

- $N_{0}=150$

- $K^{t}=150 \forall t$

- For all the experiments probability of uniform crossover is 0.8 and probability of mutation is below 0.1

From Fig. 2 and 3 one can see the varying behaviors of algorithm for different values of $\mathrm{R}$. Note that lesser values of $\mathrm{R}$ means intensity of fertility selection is more. From Fig. 4 and 5 one can see the varying behaviors of algorithms for different values of $\bar{\xi}$.

From the above simulations the emergence of $R$ and $\bar{\xi}$ as important parameters is obvious. By changing these parameters corresponding behavior of algorithms can be expected in a meaningful way. [3] gives simulation results for linearly decreasing values of $K^{t}$ in the process. $K^{t} \forall t, \mathrm{R}, \bar{\xi}$ constitute an important set of parameters for ACE-algorithms by which one can control intensities of the two important episodes of selection: viability and fertility selection.

\section{CONCLUSION}

In this paper we have introduced the concept of "two episodes of selection" in evolutionary computation by means of "A Conceptual Evolutionary model"(ACE-model). ACEmodel is based on simple and essential concepts of Darwinian evolution, and it offers abstract and structural framework for evolutionary computation. It offers relevant parameters and captures two episodes of selection as two phases of evolutionary cycle. One can construct various evolutionary algorithms by mechanizing these phases.

Our conclusions are as follows:

- selection captured in episodes offers better flexibility in bringing complex mechanisms of natural selection into evolutionary computation frameworks

- by controlling $K^{t} \forall t$ and choosing $\boldsymbol{R}$ and $\vec{\xi}$ appropriately, we can improve efficiency of evolutionary algorithms in 


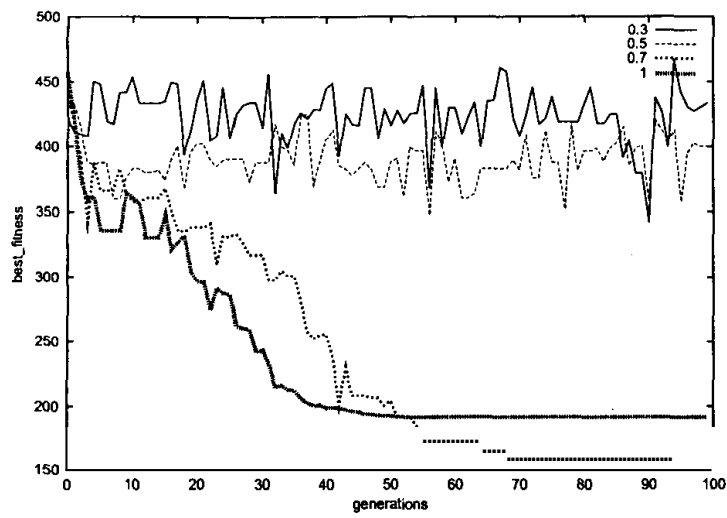

Fig. 2. Rastrigin: $\vec{\xi}=4$ for different values of $\boldsymbol{R}$

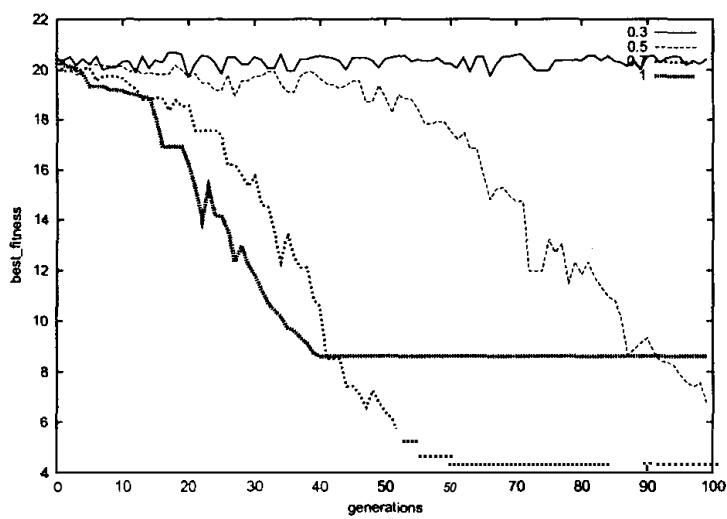

Fig. 3. Ackley: $\overline{\boldsymbol{\xi}}=\mathbf{4}$ for different values of $\mathrm{R}$

terms of convergence and quality of solutions found.

- ACE-model can be used as unified framework for evolutionary algorithms.

We have given here an abstract presentation of ACE-model and we are currently working on theoretical aspects of the proposed concepts.

\section{References}

[1] Richard Dawkins. Replicator selection and the extended phenotype. pages 125-141. Bradford Books, The MIT Press, Cambridge, Massachusetts London, England, 1984.

[2] Ambedkar Dukkipati and M. Narasimha Mrty. A conceptual evolutionary model to design novel evolutionary algorithms. In Nagib Callaos, Susana Esquivel, and Jamika Burge, editors, Proceedings of the Fifth WorldMulti-Conference on Systemics, Cybernetics and Informatics (SCI'2001), volume 3, pages 161-166. International Institute of Informatics and Systemics(IIIS), 2001.

[3] Ambedkar Dukkipati and M. Narasimha Murty. Evolutionary model based on malthusian criteria: Malthus evolutionary algorithm. In Proceedings o International Conference on Computational Intelligence, Robotics and Autonomous Systems (CIRAS'2001), pages 125-130, 2001.

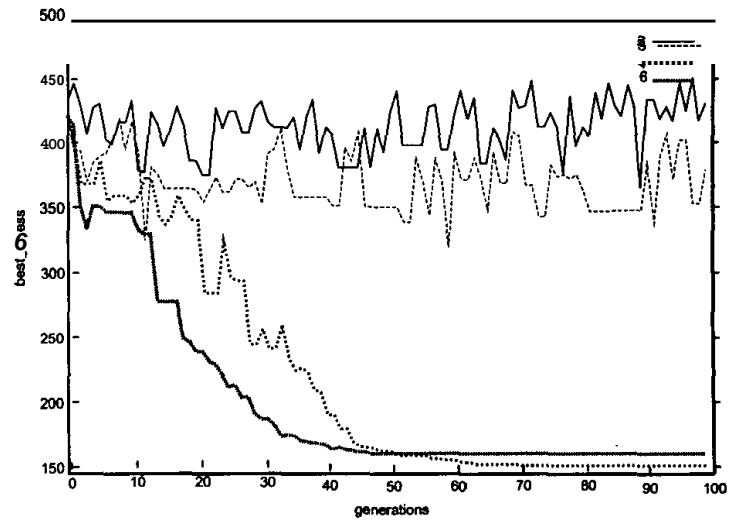

Fig. 4. Rastrigin: $\boldsymbol{R}=0.7$ for different values of $\bar{\xi}$

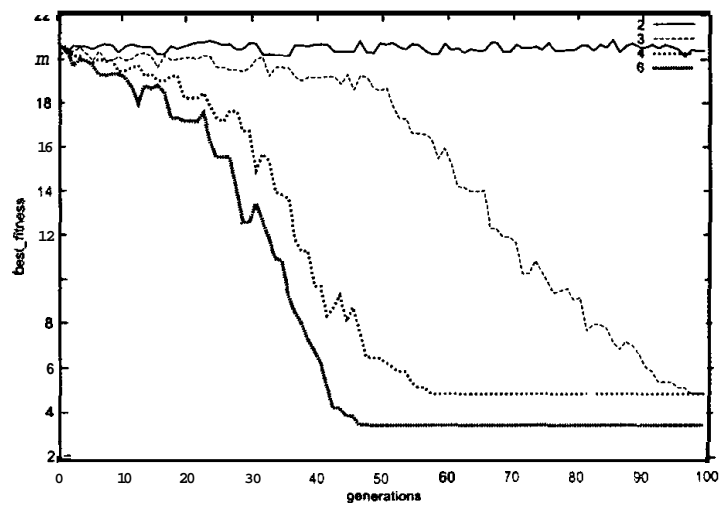

Fig. 5. Ackley: $\mathrm{R}=\boldsymbol{0} . \mathbf{7}$ for different values of $\overline{\boldsymbol{\xi}}$

[4] David B. Fogel. An introduction to simulated evolutionary optimization. IEEE Transactions on Neural Networks, 5(1):3-14, 1994.

[5] David L. Hull. Units ofevolution: A metaphysical essay. In U. J. Jensen and R. Harré, editors, The Philosophy of Evolution, Harvester Studies in Philosophy, chapter 1, pages 23-44. The Harvester Press Limited, Brighton, Sussex, 1981. General Editor: Margaret A. Boden.

[6] A Keane. An introduction to evolutionary computation in design, search and optimization. In L. Kallel, B. Naudts, and A. Rogers, editors, Theoretical Aspects of Evolutionary Computing. Springer-Verlag, Berlin Heidelberg, 2001.

[7] Barry McMullin. Essays on darwinism. 1: Ontological foundations. Technical Report bmcm920 1, School of Electronic Engineering, Dublin City University, Dublin 9, Ireland, March 1992.

[8] Heinz Muhlenbein and Dirk Schlierkamp-Voosen. Predictive models for the breeder genetic algorithm. Evolutionary Computation, 1(1):2549, 1993.

[9] Elliott Sober. The two faces of fitness. In R. Singh, D. Paul, C. Krimbas, and J. Beatty, editors, Thinking about Evolution: Historical, Philosophical, and Political Perspectives. Cambridge University Press, 2001.

[10] H Spencer. The Principles of Biology, volume 1. Williams and Norgate, London and Edinburgh, 1864.

[11] B Walsh and M Lynch. Individual fitness and the measurement of univariate selection. In Evolution and Selection of Quantitative Traits in preparation). Sinauer Associates, 2001. 